\title{
Cintigrafía renal con Tc99m DMSA (Acido dimercaptosuccínico) en pielonefritis aguda
}

\author{
Haydêe Domic T. ${ }^{1}$; Elizabeth Lagos R. ${ }^{2}$; Colomba Norero $V^{2}$ \\ Renal Tc99m DMSA scintigraphy in acute pyelonephritis
}

\begin{abstract}
Twenty patients, 6 months to 11 year old, with clinicaily recognized acute pyalonephritis, were evaluated with Tc99m DMSA renal scintigraphy in the first 15 days of their illness to validate this method in the detection of the acute renal inflamatory dissase, In $85 \%$ of Tc99m DMSA scintigrapnies some kind of abnormality (focal or diffuse, unilateral or bilateral) was observed. Second renal images obtained at an average 8.5 months later, showed tota! disappearance of abnormal findings in 42\%; partial regression in $50 \%$ and no changes in $8 \%$ of these cases. Tc99m DMSA scintigraphy seems to be a good diagnostic tool to confirm acute renaf compromise in urinary tract infections. Besides follow up examinations, at least three months later with this same method, were able to confirm sustained renal lesions.
\end{abstract}

\{Kev words: acute prelonephritis, Tc99m DMSA renal scintigraphy, infants, children.\}

La infección urinaria (ITU) es la enfermedad más común del tracto urinario en los niños. Aunque en Chile no hay estadisticas fidedignas, se sabe que es la causa de consulta rás fre. cuente en el policlínico de nefrología infantil y la tercera de hospitalizaciones pediátricas, después de las enfermedades pulmonares y djgestivas.

Las manifestaciones clínicas de JTU dependerẳn de la edad del niño. Su cuadro puede ser muy ambiguo en los menores de dos años. El diagnóstico se basa en el urocultivo y el sedjmento urinario, pero el intento de determinar su localización (infecciones altas o de pa. rénquima y bajas) puede ser dificil, ya que los examenes de laboratorio no dan certeza sobre el compromiso parenquimatoso. Las técnicas convencionales de diagnóstico por imágenes, pielografía endovenosa (PEV) y ultrasonografia (US), no han demostrado alta sensibilidad $y$ especificidad, en tal sentido, durante la fase aguda de la pielonefritis aguda (PNA) ${ }^{1-3}$.

La incorporación de la cintigrafía renal con Tc99m DMSA (ácido dimercaptosuccínico), en el estudio del compromiso parenquimatoso de la

1. Unidad de Medicina Nucleas. Hospital San Juan de Dios. Facultad de Medicina. División Occidente, Universidad de Chile.

2. Servicio y Departamento Pediatría, división Occidente, Universidad de Chile, Unidad de Nefrología Infantil, Hospital San Juan de Dios.
PNA, es un aporte de indudables ventajas. La llegada del radiofámaco por vía sistémica y su fijación en las células del túbulo proximal ${ }^{4,}{ }^{5}$, елtrega información morfológica y funcional sobre la masa renal, que puede estar alterada en la infección en forma difusa, focal o ambas, en las diferentes situaciones de đaño parenquimatoso agıdo o crónico del riñón ${ }^{6,7}$.

La detección del daño morfológico y funcional precoz por este método (que antecede a to que puede observarse en fases más tardias con otros sistemas de exploración por imágenes) facilita la observación y el manejo preventivo de pacientes potencialmente en riesgo, permite detectar tempranamente la posibilidad de reflujo vésico-ureteral (RVU) y a controlar serjadamente a los pacientes, sin riesgo de irradiación excesiva.

El objetivo de este trabajo fue estudiar la contribución de la cintigrafía renal con Tc99m DMSA para objetivar el compromiso del parénquima renal en pacientes con PNA en nuestro medio.

\section{Material y Método}

Del total de niños de ambos sexos que consultaron por PNA en el hospital San Juan de Dios entce los años 1987 y 1989 se seleccionaron los que cumplieron bs sigujentes criterios: fiebre, velocidad de sedimentación mayor o igual a $25 \mathrm{~mm}$ en la primera hora, leucocitosis mayor de 12.500 por milímetro cúbico, leu. 
cocituria sobre 10 glóbulos blancos por campo, cilindros leucocitarios o células tubulares en el sedimento de orina, urocultivo con desarrollo de algún tipo de bacteria en más de 100.000 colonias por milímetro cúbico y cintigrafía renal Tc99m DMSA realizada dentro de los primeros quince dáas de la enfermedad. En 12 de ellos se repitió la cintigrafía renal entre 15 días y 21 meses đespués ( $\overline{\mathbf{x}} 8,5$ meses) después del primer examen.

Los cintigramas renales se efectuaron con $T c 99 m$ DMSA adecuando la dosis a $\mathrm{i}$ i.dad del paciente (1 milicurie en mayores de 1 año, 700 a 800 microcuries entre 1 mes y 1 año y 500 microcuries en recién racidos), realizándose imágenes de diferentes proyecciones al menos 2 horas después de inyectado el radiofármaco. Se analizaron visualmente las imágenes analógicas y se clasificaron en las siguientes categorías: normales, cuando la distribución del sadiofámaco era homogénea heterogéneos, sì había pérdida de la homogenicidad de la distribución del DMSA sin defectos focales definidos; alteración focal si se registraba un defecto de captación del DMSA en un área bien definida de la corteza renal.

El total de la casuistica quedó constituido por 20 niños (10 hombres y 10 mujeres), con rango de edades de 6 meses a 11 años ( 10 menores de 2 años y 10 mayores).

\section{Resultados}

En el período agudo de la PNA, 85\% (17/20) de los pacientes mostraron alteraciones del cirtigrama, la distribución de las cuales se detalla en la tabla 1. Los controles mostraron regresión total (figura 1) de los defectos detectados inicialmente en $42 \%$ (5/12), parcial en 50\% (6) 12) y ninguna regresión (figura 2 ) en $8 \%(1 / 12)$ de los niños estudiados en segunda oportunidad.

Del análisis del tipo de compromiso cintigráfico detectado en el primer examen de los pacientes (tabla 1) no se pudo deducir cuáles iban a tener lesiones permanentes. No se encontró

Tabla 1

Distribución de las alteraciones cintigráficas del CR Tc99m DMSA en $17 / 20$ pacientes con PNA (fase aguda)

\begin{tabular}{lcc}
\hline & $\begin{array}{c}\text { Compromiso } \\
\text { bilateral }\end{array}$ & $\begin{array}{c}\text { Compromiso } \\
\text { unilateral }\end{array}$ \\
\hline Heterogéneo & 3 & 1 \\
Alteración focal & - & 2 \\
Heterogéneo + & 4 & 7 \\
alteración focal & 7 & 10 \\
Total & 7 & \\
\hline
\end{tabular}

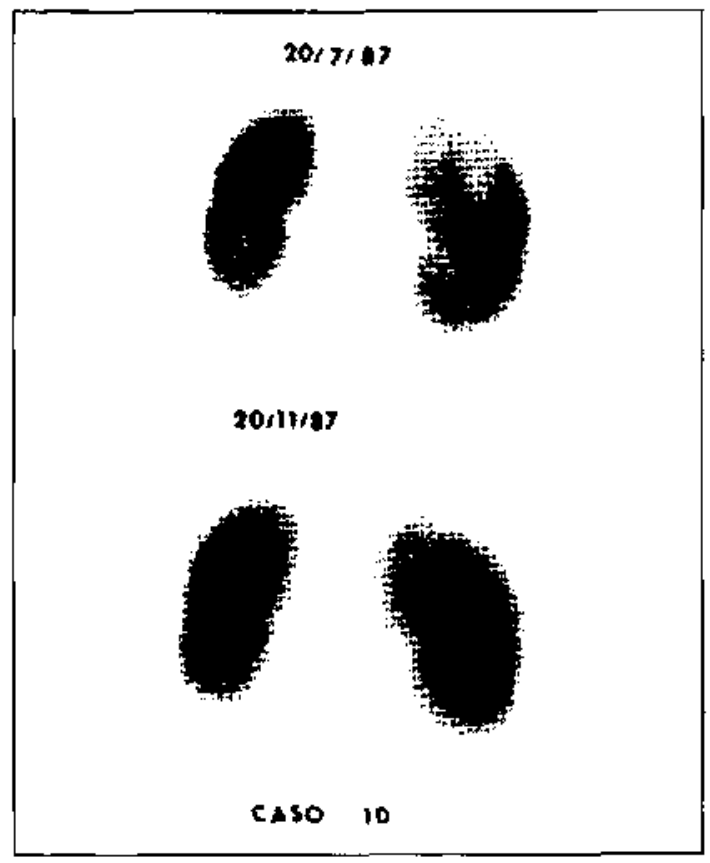

Figura 1: Paciente mujer de 9 años. Cintigrama perío. do agudo (20/7/87): distribución heterogénea $y$ defecto focal en polo superior del rinón derecho; cintigrama control $(20 / 11 / 87)$ demuestra regresión total de las lesiones.

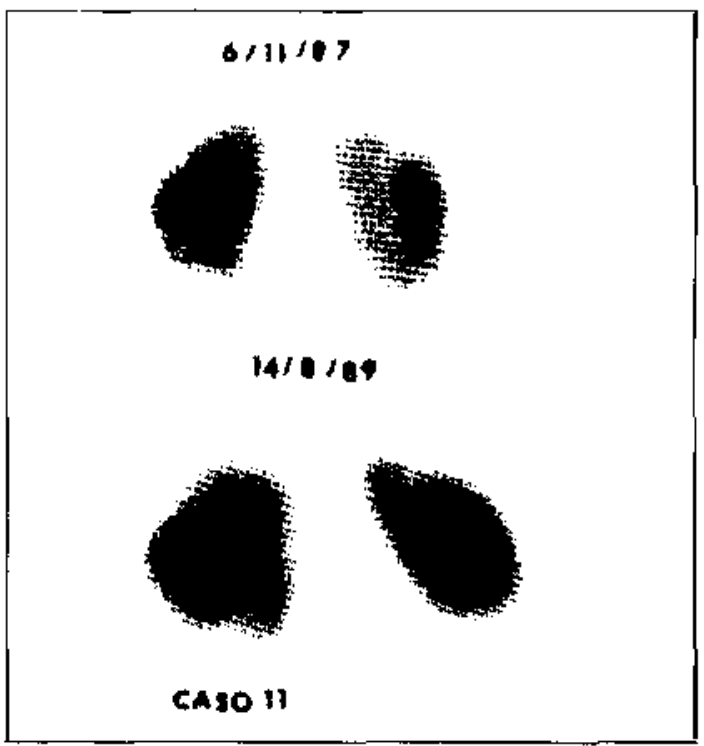

Figura 2: Paciente hombre de 1 año 11 meses, reflujo vesicoureteral bilateral. Cintigrama perfodo agudo $(6 / 11 / 87)$ : distribución muy heterogénea y defectos focales en polo infetior del rifón izquiesdo y superior derecho; cintigrama control $(14 / 8 / 89)$ persistencia de las lesiones. 
una relación estricta entre bilateralidad o heterogeneidad del compromiso y permanencia de las lesiones. Del mismo modo, la naturaleza focal de las lesiones tampoco implicó regresión posterior.

Catorce pacientes tenian PEV efectuadas en lapsos variables, 16 uretrocistografías en peŕodos libres de infección y 18 exámenes por ultrasonido realizados durante la fase aguda de la enfermedad, to que se resume en la tabla 2 .

Al analizar los 7 pacientes que no demostraron regresión total de las alteraciones cintigráficas en el control se aprecia que en 5 había alteraciones subyacentes: dos tenian RVU, uno doble sistema, otro riñón en herradura $y$ uno leve ectasia derecha en la pielografía. Cabe destacar que el paciente ll tenía reflujo grado
IV y antecedente de ITU en ta anamnesis, por lo que es probable que ya tuviese daño renal previo al estudio actual. Por otra parte, dos pacientes en que no hubo regresión total en el control tenían PEV, UCG y US normales (casos 7 y 8). En ellos es probable que la infección fuese la primera, ya que coinciden con los ninos de menor edad de la casuística estudiada.

En 3 pacientes $(15 \%)$ la uretrocistografía demostró la presencia de reflujo de grado variable. Se trataba de nifios mayores (casos $3,5 y$ 11) en que otros métodos de imagenes no demostraban lesiones crónicas. Cabe destacar especialnente el caso 3 en que los US y la cintigrafia fueron normales, a pesar de tener reflujo vesicoure teral bilateral.

Tabla 2

Resultados de la exploración con métodos convencionales por inázenes en 20 niños con piejonefritis aguda $y$ cintigrafías iniciales y de segujmiento con $\mathrm{Tc} 99 \mathrm{~m}$ DMSA

\begin{tabular}{|c|c|c|c|c|c|c|}
\hline Sexo & Edad & US & PEV & UCG & C. renal & Control \\
\hline 1. $\mathbf{M}$ & $8 \mathrm{~m}$ & Nomnal & $\mathrm{No}$ & No & Normal & No \\
\hline 2. $F$ & $5 \mathrm{a}$ & Nomal & No & No & Normal & No \\
\hline 3. $M$ & $18 \mathrm{~m}$ & Normal & No & RVU II bilat. & Normal & No \\
\hline 4. $F$ & $5 \mathrm{a}$ & Nomnal & Doble sist RD & Normal & Alt. $H+F$ en RD & $\mathrm{RP}$ \\
\hline 5. F & $5 a$ & $\begin{array}{l}\text { Netromegalia } \\
\text { bilateral }\end{array}$ & No & $\begin{array}{l}\text { RVU II det. } \\
\text { ¿doble sist.? }\end{array}$ & Alt, $\mathbf{H}+\mathrm{F}$ en $\mathrm{RD}$ & $\mathbf{R} \mathbf{P}$ \\
\hline 6. $M$ & $1 \mathrm{a}$ & Normal & $\begin{array}{l}\text { Leve pielo- } \\
\text { ectasia RD }\end{array}$ & Nomal & Alt.H + F en RD & $\mathbf{R P}$ \\
\hline 7. $\mathrm{M}$ & $9 \mathrm{~m}$ & Normal & Normal & Normal & Alt. $\mathrm{H}+\mathrm{F}$ en $\mathrm{RI}$ & RP \\
\hline 8. M & $6 \mathrm{~m}$ & Niormal & Normal & Normal & Alt. $\mathrm{H}+\mathrm{F}$ bilat. & $\mathbf{R P}$ \\
\hline 9. $\mathrm{I}^{\mathrm{T}}$ & $7 \mathrm{a}$ & R. herradura & $\begin{array}{l}\text { Leve dilatac. } \\
\text { sistema izq. }\end{array}$ & Nomal & Alt. $\mathrm{H}+\Gamma$ bilat. & $\mathbf{R P}$ \\
\hline 10. $F$ & 9 a & $\begin{array}{l}<\text { ecogen. polo } \\
\text { inf. } R D\end{array}$ & Normal & Normal & $\begin{array}{l}\text { Alt. } H+F \text { polo } \\
\text { sup. } R D\end{array}$ & $\mathrm{RT}$ \\
\hline 11. $\mathrm{M}$ & $23 \mathrm{~m}$ & No & $\begin{array}{l}\text { S. pielocali- } \\
\text { ciario y uréter } \\
\text { der. dilatac. } \\
\text { leve a moderada }\end{array}$ & $\begin{array}{l}\text { RVU IV der. } \\
\text { RVU III izq. }\end{array}$ & $\begin{array}{l}\text { Alt. } H+F \text { en } R D \\
\text { Alt. } H \text { en } R I\end{array}$ & SR \\
\hline 12. F & $6 \mathrm{~m}$ & Normal & Normal & No & $\begin{array}{l}\text { Alt. } H \text { en RD } \\
\text { Alt. } H \text { en } R \mathbf{l}\end{array}$ & RT \\
\hline 13. M & $19 \mathrm{~m}$ & Normal & Normal & Normal & Alt. $H$ en RD & RI \\
\hline 14. $F$ & 5 a & Normal & Normal & Normal & Alt. $\mathrm{H}+\mathrm{F}$ en $\mathrm{RD}$ & RT \\
\hline 15. F & $7 a$ & Normal & $\begin{array}{l}\text { Probables } \\
\text { cálculos }\end{array}$ & Normal & Alt. $\mathrm{H}$ bilat. & No \\
\hline 16. M & $2 \mathrm{~m}$ & Nomal & $\downarrow$ tamaño RD & Normal & $\begin{array}{l}\text { Alt, } H \text { en RD } \\
\text { Alt. } H+F \text { en RI }\end{array}$ & No \\
\hline 17. $\mathrm{F}$ & $14 \mathrm{~m}$ & Normal & Normal & Normal & Alt. F en RD & No \\
\hline 1B, F & 11 a & Normal & No & No & Alt. $\mathrm{H}+\mathrm{F}$ en $\mathrm{RL}$ & No \\
\hline 19. M & $9 \mathrm{~m}$ & No & $\begin{array}{l}\text { Leve piclo- } \\
\text { ectasia RI }\end{array}$ & Normal & Alt. F en RD & RT \\
\hline 20. $\mathbf{M}$ & $5 \mathrm{a}$ & Nommal & No & Normal & Alt. $H+F$ en $R D$ & No \\
\hline
\end{tabular}

US ultrasonidos. UCG: uretrocistografía radiológica. PlV: pielografía de eliminación. C. renal: cintigrafía renal Tc99m DMSA. No: no se realizó el examen. Alt. H: alteración heterogénea. Alt. $F$ : alteración focal. Alt. H + F: alteración heterogénea y focal. Ni normal. RT: regresión total. RP: regresión parcial, \$R: sin regresión. RD: rịnón derecho. RI: rifón izquierdo. Bilat,: bilateral. Det,: derecho. Izq. : izquierdo. 


\section{Comentario}

E1 DMSA es una sustancia quelante (PM 182). Por esta propiedad es capaz de unirse al tecnecio 99 metaestable ( $\mathrm{T} 09 \mathrm{~m}$ ) y formar un complejo Tc99m DMSA con gran capacidad de unirse a proteînas plasmáticas, especialmente seroalbúmina, por lo que es filtrado en bajo porcentaje. Este complejo se fija a las células del túbulo proximal y primeras porciones del asa de Henle, probablemente a una metaloproterna del citoplasma. La forma exacta en que se incorpora el radiofármaco a la célula tubular no está completamente dilucidada. Se invoca especialmente un mecanismo que involucta su lle. gada por la circulación peritubular hacia la célula blanco. En procesos infecciosos en que se produce edema peritubular con aumento de la presión intersticial se altera de algún modo el flujo regional $y$, por ende, la distribución intrarrenal del DMSA. Además se han serialado al. teraciones a nivel de la membrana ce]ular, ya sea por toxicidad directa sobre ella por el proceso infeccioso o por algún proceso metabólico que impida su transporte a través de ella ${ }^{6,10}$.

En un estudio realizado en cerdos, a los cuales se les provocó RVU e infección del tracto urinario, se realizaron cintigrafías con Tc99m DMSA, sacrificando luego a los animales para comparar las alteraciones anatomopatológicas y las encontradas en el cintigrama. Nunca se observaron defectos cintigráficos en ausencia de cambios histológicos y sjempre se encontraron alteraciones en la anatomía patológica cuando existian en el cintigrama. Sin embargo, pequeñas lesiones histológicas no se evidenciaron en el cintigra$\mathrm{ma}^{11}$.

Sólo tenemos conocimiento de un estudio sistemático de la evaluación del cintigrama renal con DMSA en el điagnóstico diferencial entre ITU alta y baja ${ }^{\circ}$, con control evolutivo post-tratamiento. De un total de 24 niños se llegó al diagnóstico de ITU en 15 y baja en 9, utilizando los criterios de Pylkkänen ${ }^{15}$. De los que tenian ITU alta, $12 / 15(85 \%)$ y $1 / 9$ de los con ITU baja presentaron alteraciones en el cintigrama. El cintigrama control sólo se normalizó en $6(50 \%)$. Un casu de 9 con ITU baja mostró alteración en el cintigrama, pero resultó ser un quiste al explorar con US, lo que demuestra la importancia de complementar las diferentes técnicas de diagnóstico por imágenes. Las ci. fras del mencionado trabajo son coincidentes con nuestra experiencia, en que se analizaron sólo PNA.

La regresión de las alteraciones cintigráficas del período agudo ha sido observada también por Hanmaker ${ }^{12}$ y Veber y Moller ${ }^{13}$, en cifras variables que en esta última comunicación son de 20\% a dos años promedio del primer examen. En nuestro trabajo $4 / 12$ pacientes (33\%) con cintigrama repetido en un promedio de 8 meses, mostraron regresión total de las lesiones. Parece evidente que, para evaluar adecuadamente la regresión, el control debe ser efectuado después de 3 meses del episodio inicial, para permitir la resolución de las lesiones parenquimatosas. En uno de los primeros pacientes de nuestra serie efectuamos el control a los 15 días, encontrando sólo regresión parcial de las lesiones. No se ha controlado posteriormente. Creemos que después de $3 \mathrm{me}$. ses del episodio agudo, el cintigrama Tc99m DMSA puede detectar con seguridad las lesiones permanentes de un episodio agudo, cicatrjces que sólo se aprecian a la IVP entre 8 meses a 2 años después ${ }^{7,14}$.

Aunque el objetivo de nuestro trabajo no fue comparar los distintos métodos de exploración por imágenes en su contribución al diagnóstico de la PNA, queremos destacar que la PEV y el US no muestran una sensibilidad adecuada para evidenciar el compromiso parenquimatoso en la fase aguda de la enfermedad, tal como se ha ser̃alado repetidamente ${ }^{\mathrm{B}, \boldsymbol{9}} \mathrm{y}$, por lo tanto, no contribuyen al diagnóstico diferencial entre ITU alta y baja. Esto se ve avalado en nuestra casujstica, en que los pacientes con PNA presentan exclusivamente alteraciones cintigráficas, sin existir compromiso por PEV, US y UCG.

Sin embargo, la PEV es un excelente método para el estudio morfológico del tracto urinario, requiriendo para ello de función renal adecuada. Asi también en nuestros casos la PEV demostró en 2 pacientes alteraciones morfológicas: un riñón en herradura y un doble sistema. En otros 3 pacientes se aprecia leve ectasia excretora que se puede interpretar como indice indirecto de infección actual.

Las US tienen gran valor en la detección de cicatrices renales. En la etapa aguda demuestran alteraciones más focales como abscesos, pionefrosis y colecciones perirrenales. En nuestros casos la US permitió confirmar el tifión en herradura y plantear una nefromegalia bilateral (en un paciente con RVU). En ninguno de nuestros 
enfermos se detectaron cicatrices renales con este método.

Sin duda que la tomografía axial computarizada es una técnica de alta sensibilidad en la evaluación del compromiso renal de la infección aguda, pero el alto costo y la dosis de irradiación no justifican sI uso rutinario en la población pediátrica'.

Actualmente la recomendación práctica ante un niño con infección urinaria es efectuar ultrasonidos y uretrocistografía para descartar la presencia de reflujo vesicoureteral y alteración parenquimatosa renal. Indudablemente la incorporación de la cintigrafía con Tc99m DMSA contribuye a la detección del compromiso parenquimatoso alto, aclarando el diagnóstico de PNA. Su reiteración con fines de control, efectuada después de tres meses, puede establecer la presencia de compromiso renal permanente, planteando una conducta de observación mantenida de los pacientes afectados.

Si bien no todos los servicios hospitalarios cuentan con este método, es altamente probable que vaya a ser considerado de rutina en pocos años. Debe destacarse que no se plantea como método diagnóstico único, sino como complementario a los actualmente utilizados, ofrecjendo ventajas en cuanto a confirmación del diagnóstico de compromiso parenquimatoso agudo y de detección temprana de lesiones renales permanentes.

\section{Resumen}

Veinte pacientes de 6 meses a 11 anos de edad, con diagnóstico clínico de pielonefritis aguda, fueron evaluados con cintigrafia renal Tc99m DMSA en los primeros 15 días de su enfermedad, a fin de validar dicho método en la detección del compromiso agudo del parénquima renal por el proceso inflamatorio. Se comprobó alteración en la captación resal del radiofármaco en $85 \%$, en forma focal o difusa, uni o bilateral. Un segundo cintigrama efectuado en promedio 8,5 meses después del inicial, mostró regresión total de las lesiones en $42 \%$; parcial en $50 \%$ y ninguna regresión en $8 \%$ de los casos. Se con. cluye que el cintigrama renal $\mathrm{T} c 99 \mathrm{~m}$ DMSA es un buen método diagnóstico para detectar compromiso parenquimatoso agudo en la infección urinaria. Su control después de tres meses puede informar sobre la persistencia de las lesiones.
(Palabras clave: infección urinaria alta, pielonefritis aguda, compromiso renal, cintigrafia renal, Tc99m DMSA.)

\section{Referencias}

1. Kullendorff. C.M.; Laurin, S.; White, T.: Evaluation of renal parenchyma in chikdren by DMSA scintigraphy, $X$-ray computed tomography and intravenus urography, Acta Paediatr Scand 1987, 76: $284-287$.

2. Sty, J.R.; Wells, R.G.; Brice, A.: Schroeder; Srershack, R.J.: Diagnostic imaging in paediatric renal inflamatory disease. JAMA, 1986, 256: 895899.

3. Lebouitz, R.L.: Mandell, $J_{\text {: }}$ Urinary tract infection in children: putting radiology in its place. Radiology 198?, 165:1-9.

4. Chorv, L.R.i Bloutox, M.D.: Renal sadiopharmaceuticals. An update. Sen Nucl Med, 1982, XII: 227-231.

5. Kowalsky, R.S.: Perty, R.J. Radiopharmaceuticals in Nuclear Medicine Practice Series Ed. Sheldon Baum M.D.; Ed. Appleton and Lange. Norwalk, Conneticut, Los Altos, California 1987: 327334.

6. Gordon, I. Indications for Tc99m-DMSA scan in children. J Urol 1987, $137: 464-467$.

7. Verber, I. $a_{*}$; Strudley, M.R.: Meller, S.T.: Tc99mDMSA scan as first investigation of urinary tract infection. Arch Dis Child 1988, 63: 1320-1325.

8. Godiraich, N.P.; Ramos, O.l.; Goldraich, I.H.: Urography versus DMSA scan in children $u$ 'ith vesicoureteric reflux. Pediatr Nephrol 1989 , 3: 1.5 .

9. Smalle, J.M. The DMSA scan and intravenuus urography in the detection of renal scarring. Pedjatr Nephrol 1989, 3: 6-8.

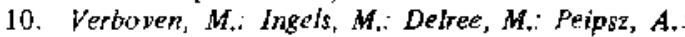
Tc99m-DMSA scintigraphy in acute urinary tract infection in children. Comunicación personal, 1989.

11. Parkhouse, H.F.; (rodley, M.l., Cooper, J.: Ridson, R.A. and Ronslev, P.G.: Renal imaging with Tc99m-DMSA in detection of acute pyelonephritis: an experimental study in the pig. Nucl Med Comm $1989,10: 63-70$.

12. Hardmaker, $H$.: Nuclear renal imaging in acute pyelonephritis. Sem Nucl Med 1982, XII: 246253.

13. Verber, I.G.: Meller, S.T.: Serial $99 \mathrm{~m}$ Tc-DMSA scans after urinary infections presenting before the age of 5 years. Atch Dis Child, 1989, 64, 1533-1537.

14. Merrick, M.V.; Uttley, W.S.: Wild, M.B.: The detection of pyelonephritis scarring in children by radioisotopes imaging. Brit J Rad 1980, 53: 544556 .

15. Pylkkänen, $J_{.:}$VIIska, $J_{.:}$Koskimies, $O .:$ The value of level diagnosis of childhood urinary tract infection in predicting renal injury. Acta Paediatr Scand, $1981,70: 879-883$. 